\section{How do Creativity Levels and Stimulus Types Matter? A Preliminary Investigation of Designer Visual Association}

\section{Abstract}

This study aimed to examine the brain activations of designers during visual association and identify the differences in brain activations caused by distinct visual stimulus types among designers with different creativity levels. Twenty-one professional production designers were recruited and divided into three groups. The top third and bottom third of the participants ( 7 for each) were divided into high creativity (HC) and low creativity (LC) groups for neural comparison analyses. The derived results are outlined as follows: (i) The brain activations of the production designers notably increased in the prefrontal and parietal regions during visual association; (ii) the spectral power of most HC designers was lower than that of the LC designers; (iii) realist art stimulation evoked strong activation in the anterior ventral regions, whereas abstract art stimulation primarily activated the posterior regions; (iv) the differences in brain activations between the $\mathrm{HC}$ and LC designers resulting from realist art stimulation were generally larger than were those resulting from abstract art stimulation; and ( $v$ ) the brain activations of the HC designers resulting from abstract art stimulation were stronger than were those resulting from realist art stimulation, whereas an opposite trend was observed in the LC designers. Few studies to date have empirically explored the relationship between the creativity levels of designers and their visual association. Enhancing the creative performance of designers should feature among the primary goals of the design industry and design education. This study elucidates a novel approach in this critical research area, although further studies are necessary.

Keywords: Creativity; Designer; Electroencephalography; Stimulus types; Visual association

\section{Yu-Cheng Liu, Yu-Hsuan Sylvia Yang and Chaoyun Liang}

Department of Bio-Industry Communication and Development, National Taiwan University, Taipei, Taiwan

\section{Corresponding author: Chaoyun Liang}

” cliang@ntu.edu.tw

Department of Bio-Industry Communication and Development, National Taiwan University, No. 1, Sec. 4, Roosevelt Road, Taipei, 10617, Taiwan.

Citation: Liu Y, Yang YS, Liang C. How do Creativity Levels and Stimulus Types Matter? A Preliminary Investigation of Designer Visual Association. J Neurol Neurosci. 2017, 8:2.

Received: March 29, 2017; Accepted: April 14, 2017; Published: April 17, 2017

\section{Introduction}

Creativity is implemented and transformed into commercial value through the design process. There is no potential for commercial innovation if design lacks creativity. Because of the requirement of the design profession, each designer is endowed with unique talents and engages in distinctive cognitive processes. Crilly [1] thus suggested that professional performance differs among designers depending on their levels of creativity. Unlike psychology and management, limited research is available regarding creativity within the field of design. Designing is a dynamic process in which the hand, eye, and mind collaborate [2]. Understanding the cognitive processes used in creative practices is essential to design research. However, research on the neurocognitive basis of design is limited to date. Moreover, studies on characterizing or recognizing design as a distinct cognitive phenomenon are not available [3].

Designers are typically sensitive to visual stimuli and seek a diverse range of visual references while creating a product. Goldschmidt [4] observed that a sensitive designer possesses a prepared eye, which can respond to the stimuli it encounters in any environment. Goldschmidt emphasized that visual stimuli are ubiquitous and their exploitation is serendipitous. Visual association, the ability to meaningfully relate visual stimuli to other previously learnt images, is significantly associated with artistic creativity [5]. Through visual association, designers can determine which target objects resemble remembered objects and subsequent creations. However, extant research on the 
visual association of designers is limited [3]. Liang and colleagues [6] warned that overlooking scientific evidence of cognitive reactions can engender erroneous conclusions regarding the relationships between visual activities and design creativity.

Numerous studies have explored visual association in the brain $[3,7,8]$. The present study represents an additional effort to investigate the brain activations of visual association in production designers through electroencephalography (EEG). In addition, we analyzed the differences in brain activations between highcreativity $(\mathrm{HC})$ and low-creativity $(\mathrm{LC})$ designers as well as the differences in brain activations resulting from abstract and realist art stimulations. The research questions are listed as follows: (i) Which brain regions are relatively active when production designers engage in tasks involving visual association? (ii) What are the differences in brain activations caused by two distinct types of pictorial representation (i.e., realism and abstraction) when designers engage in these visual association tasks? (iii) What are the differences in brain activations between $\mathrm{HC}$ and LC designers when they engage in experimental tasks?.

\section{Neurocognitive remarks on visual stimulation, visual association, and visual creativity}

Designers often seek external sensory representations as sources of inspiration and simultaneously use internal mental imagery to form design resolutions and acquire design expertise [9]. Scholars have stated that abstract stimuli are beneficial for generating creative ideas and overcoming design fixation $[10,11]$. However, Goldschmidt and Smolkov [12] suggested that the influence of visual stimuli on designer performance mainly depends on the type of design problem. Previous neurocognitive studies have indicated that the prefrontal cortex plays a key role in aesthetic perception $[13,14]$ and that brain activation generally increases along a gradient of posterior (lower-tier) to anterior (higher-tier) ventral visual regions with increased object resolution $[15,16]$, which implies that different types of pictorial representations exert distinct effects on aesthetic stimulation. However, prior research investigating this topic has drawn inconsistent conclusions. For example, Fairhall and Ishai [15] observed stronger activation in the higher-tier visual regions and the temporoparietal junction when participants received realist stimuli than when they received abstract or surrealist stimuli, whereas Silveira and colleagues [14] indicated that viewing realist paintings elicits significantly higher activation in the visual cortex and in the precuneus.

Regarding visual association, Andreasen [17] observed activations in V1, the bilateral middle and inferior frontal gyri, the bilateral fusiform gyri, and the left and right angular gyri when participants, particularly creative individuals, generated visual associations. Visual association is related to visual retrieval and depends on mnemonic mechanisms in the temporal cortex $[18,19]$. In addition, the default mode network (DMN) is active when people engage in internally focused tasks such as visual association, future imaginings, and mental simulations $[20,21]$. Moreover, the medial temporal cortex stores memories and associations from prior experiences, and the medial prefrontal cortex facilitates the construction of mental simulation [20]. Such goal-directed simulation requires the engagement of the DMN, executive dorsolateral prefrontal cortex, and distributed network, including the medial prefrontal cortex, medial temporal, and parietal regions [22]. In particular, the neural correlates of simulations of future events can be observed in the medial prefrontal, posterior cingulate, temporoparietal, and medial temporal cortices [23].

With regard to visual creativity, prior research has provided consistent evidence supporting low frequency ranges. For example, strong delta coherence has been observed in the occipitotemporal regions of $\mathrm{HC}$ individuals [24,25]. A similar pattern has been demonstrated in the theta band of $\mathrm{HC}$ participants $[25,26]$. Low alpha coherence has been found to be activated in the bilateral frontal and middle parietal regions of creative individuals $[26,27]$. Substantial alpha synchronization has been found in the right posterior parietal regions during the generation of alternatives and improvisation tasks [28]. In addition, Sviderskaya [29] reported a greater increase in the brain wave coherence in the right frontal and occipital regions of artists than in those of novices. Gruzelier [30] indicated that mediation between distal and widely distributed connections underpins the integrative attributes of alpha-theta training in creative performance enhancement, long-distance functional connectivity, and psychological integration. Moreover, when alpha-theta neurofeedback was consistently trained, the commitment, confidence, emotional expression, and enjoyment of musicians, dancers, and actors was enhanced during their performances [31].

\section{Methods}

\section{Participants}

In this study, 21 healthy production designers were invited to participate in an EEG experiment. The participants were required to meet the following inclusion criteria: (i) more than 10 years of work experience in the industry of production design, (ii) receipt of awards in international competitions for production design, and (iii) availability during July and August 2016. Among the participants, 7 were women and 14 were men, and the age range was 37 to 48 years. The participants were guided to fill out a creative personality scale [32] questionnaire before the experiment began. The CPS questionnaire contains 18 positive and 12 negative items. One point was added for selecting a positive item, and 1 point was subtracted for selecting a negative item. Because higher CPS total scores indicate higher creativity, the top third and bottom third of the participants ( 7 for each) were divided into $\mathrm{HC}$ and LC groups for neural comparison analyses.

\section{Materials}

The visual stimuli used in this study were artworks by JeanFrançois Millet (representing realism) and Pablo Picasso (representing abstraction). In addition to representing distinct art movements, the majority of these artworks have entered the public domain in most countries because they were published 
prior to 1930 . The researchers first nominated 10 representative pieces of each artist's work individually and then compared the selections to ensure that the same work did not appear twice. The artworks were also compared on the basis of their characteristics such as clarity, composition, repetition, and figure-ground contrast. After several rounds of comparison and discussion, the researchers agreed on a list of six artworks by each artist, thereby selecting a total of 12 paintings for use as visual stimuli. The six artworks by each artist were then randomly presented to the participants during the experiment.

A 32-channel wireless EEG cap BR32S headset, developed by Brain Rhythm Inc., Taiwan, was used in this experiment. This wearable system has 16-bit quantization and a sampling rate of $250 \mathrm{~Hz}$. The scalp markers are placed according to the international 10-20 system with underlying cerebral structures. This headset features spring-loaded dry electrodes and a soft cap, which render it precise and convenient. The dry sensors are resilient and can be used repeatedly on hairy sites without conductive gel. The EEG data are wirelessly received via Bluetooth without external devices. Data collected from the experiments were exported in ASCII (.txt) format for analysis.

\section{Experimental protocol}

After the participants signed consent forms, the researchers recorded their brain responses during resting periods to provide a baseline. The participants were asked to verbally describe an ongoing design project or a design project that they had completed within the past 6 months. This description took approximately 120 seconds and the narrated information regarding the design problem, purpose, and imagined outcomes was recorded. Each of the 12 paintings was then displayed randomly for 60 seconds, during which the participants were asked to silently respond to the question, 'what aspect of this painting can you associate with the project just mentioned?' Subsequently, the participants verbalized their answers for 120 seconds. The EEG signals were recorded during the sessions of 60-second silence. The sessions of 120 -second verbalization were tape-recorded to help the researchers in understanding the participants' associations. The sessions were also treated as intertrial intervals to avoid recording overlapping brain responses. In total, the experiment lasted for approximately 45 minutes, including the time of project description and EEG headset testing. The process was identical for all participants to ensure the consistency of the investigation.

\section{Data analyses}

The EEG data were initially split into 1.6-second signals. A lowpass filter with a cut-off frequency of $50 \mathrm{~Hz}$ and a high-pass filter with a cut-off frequency of $1 \mathrm{~Hz}$ were applied to all 1.6-second EEG signals based on FIR filters to remove line noise and direct current drift. Channels exhibiting abnormal waveforms were removed manually and replaced by averaging data. The filtered EEG signals were then decomposed using independent component analysis (ICA) by employing EEGLAB [33]. In ICA, all components were grouped into several clusters according to the similarity of outcomes using the k-means clustering method. In this study, the scalp topography of each independent component was used to plot the three-dimensional (3D) location of an equivalent dipole through the DIPFIT function. Apart from one outlier cluster, timedomain data were then transformed into frequency-domain data by using the fast Fourier transform function. Furthermore, a paired-sample Wilcoxon signed-rank test was applied to test the differences in brain activity spectra of the $\mathrm{HC}$ and LC designers, as well as between realist art and abstract art stimulations.

According to prior research [34-36], the spectra of EEG signals were separated into five frequency bands, namely delta (0.5-3.5 $\mathrm{Hz}$ ), theta $(3.5-8 \mathrm{~Hz}$ ), alpha (range $8-13 \mathrm{~Hz}$; low, 8-9 Hz; middle, 9-11 Hz; high, $11-13 \mathrm{~Hz}$ ), beta (range $13-30 \mathrm{~Hz}$; low, $13-16 \mathrm{~Hz}$; middle, 16-20 Hz; high, 20-30 Hz), and gamma (range 30-100 $\mathrm{Hz}$; low, 30-60 Hz; typical, $40 \mathrm{~Hz}$; high $>60 \mathrm{~Hz}$ ). Only low gamma bands ranging between 30 and $50 \mathrm{~Hz}$ were investigated in the present study because high gamma bands rarely appear in EEG results.

\section{Results}

In this section, the scalp topography of the root cluster is described first, followed by the topographies of the major component clusters and their dipole plots. The colours of scalp topographies indicate brain activity, with warm colours signifying more activation and cold colours signifying less activation. By using the results of the Wilcoxon signed-rank test, we elucidate the differences in spectral power between the HC and LC designers and between realist art and abstract art stimulations. The significance levels of the null hypothesis at different frequencies are shown as red dots in the plots of the spectra.

According to the scalp map for the root cluster, both the $\mathrm{HC}$ and LC designers had relatively high brain activations in the right prefrontal and middle frontoparietal cortices when they engaged in the task of visual association with Millet's realist artworks (Figure 1a). The scalp maps and 3D dipole plots displayed in Figures $\mathbf{1 b}-\mathbf{1}$ e reveal that these brain activations could be divided into four major component clusters, namely the right prefrontal, right frontotemporal, left frontoparietal, and middle frontoparietal cortices. Notably, the areas displayed in Figures 1c-1e are critical regions forming the anterior cingulate cortex (ACC). The 3D dipole plots indicate the equivalent dipole source locations with means and their projections onto average brain images.

The results also indicate that the spectral power of the $\mathrm{HC}$ designers was lower than that of the LC designers. In the right prefrontal cluster, significant power differences were observed in the alpha, beta, and gamma bands (Figure 2a). The largest differences appeared in the low gamma band at $33 \mathrm{~Hz}$ and 43 $\mathrm{Hz}(p=0.013252)$. In the right frontotemporal cluster, significant power differences were observed in the delta, theta, beta, and low gamma bands (Figure $\mathbf{2 b}$ ). The largest differences appeared in the low gamma band at $32 \mathrm{~Hz}$ and $35 \mathrm{~Hz}$ ( $p=0.001757)$. In addition, in the left frontoparietal cluster, significant power differences were observed in the distributed delta, theta, alpha, beta, and low gamma bands (Figure $2 \mathrm{c}$ ). The largest differences appeared in the high beta band at $24 \mathrm{~Hz}(p=0.000794)$ and theta band at $5 \mathrm{~Hz}(p=0.001268)$. Finally, in the middle frontoparietal 


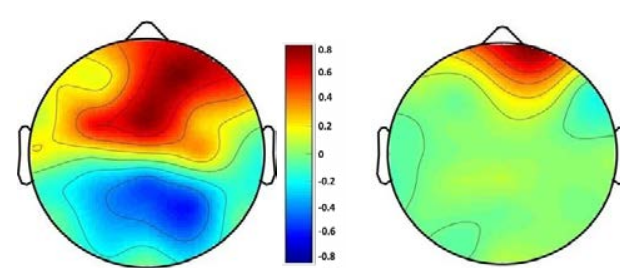

1a. Root cluster
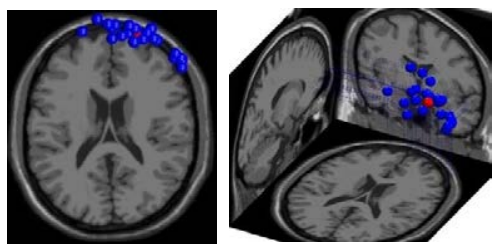

1b. Right prefrontal cluster
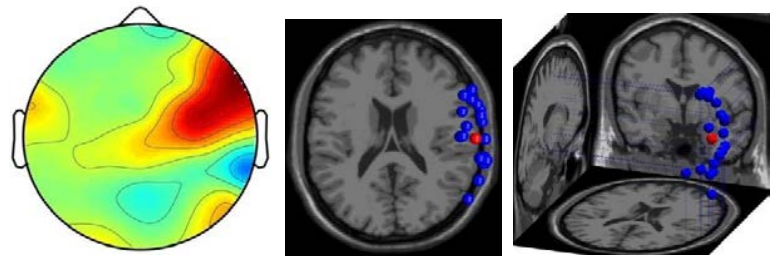

1c. Right frontotemporal cluster
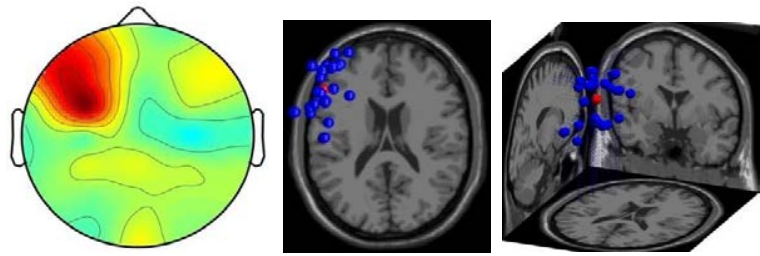

1d. Left frontoparietal cluster
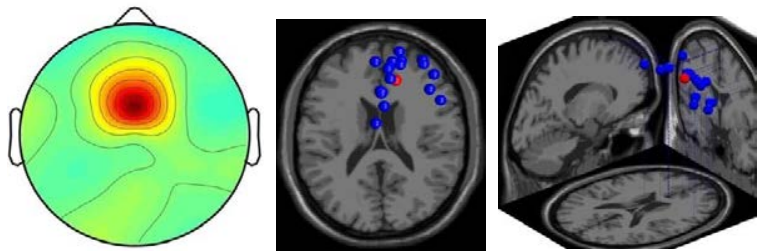

1e. Middle frontoparietal cluster

Figure 1 Scalp maps and 3D dipole plots for the realist art stimulation task (1a: scalp map for the root cluster; (1b-1e): scalp maps for the component clusters and their 3D dipole source locations).

cluster, significant power differences were observed in the alpha, beta, and low gamma bands (Figure $\mathbf{2 d}$ ). The largest differences appeared in the low gamma band at $46 \mathrm{~Hz}(p=0.001744)$ and 47 $\mathrm{Hz}(p=0.002169)$.

Both the $\mathrm{HC}$ and LC designers had high brain activations in the right prefrontal and frontoparietal cortices when they engaged in the task of visual association with Picasso's abstract artworks (Figure 3a). The scalp maps and 3D dipole plots depicted in Figures $\mathbf{3 b - 3 e}$ reveal that these brain activations could be separated into four major component clusters: the right prefrontal, right parietal, left frontoparietal, and left occipital cortices. The regions displayed in Figures $\mathbf{3 c}$ and $\mathbf{3 d}$ are markedly associated with the DMN.

Besides the right parietal cluster, our results revealed that the spectral power of the $\mathrm{HC}$ designers was generally lower than that of the LC designers. As depicted in Figure 4a (the right prefrontal cluster), only one significant power difference was observed in the beta band at $20 \mathrm{~Hz}(p=0.03203)$. In the right parietal cluster (Figure 4b), no significant power difference was observed. In the left frontoparietal cluster, two significant power differences in the beta and gamma bands were observed (Figure 4c). They appeared in the high beta band at $20 \mathrm{~Hz}(p=0.007257)$ and low gamma band at $32 \mathrm{~Hz}(p=0.041738)$. Finally, in the left occipital cluster, significant power differences were observed in the beta and low gamma bands (Figure $\mathbf{4 d}$ ). The largest differences appeared in the low gamma band at $36 \mathrm{~Hz}(p=0.000356), 32 \mathrm{~Hz}$ ( $p=0.00049)$, and $41 \mathrm{~Hz}(p=0.001643)$.

The $\mathrm{HC}$ designers had high brain activations in the right prefrontal and frontoparietal cortices when they engaged in the task of visual association (Figure 5a). The scalp maps and 3D dipole plots displayed in Figures $\mathbf{5 b} \mathbf{b} \mathbf{5 e}$ reveal that these brain activations could be separated into four major component clusters: the right prefrontal, left frontotemporal, middle parietal, and right temporal cortices. The DMN and posterior cingulate cortex are located in the middle parietal region.

Besides the right parietal cluster, our results revealed that the spectral power upon stimulation with abstract art was generally higher than that upon stimulation with realist art. In the right prefrontal cluster (Figure 6a), only one significant power difference was observed in the theta band at $7 \mathrm{~Hz}(p=0.045541)$. In the left frontotemporal cluster, significant power differences 


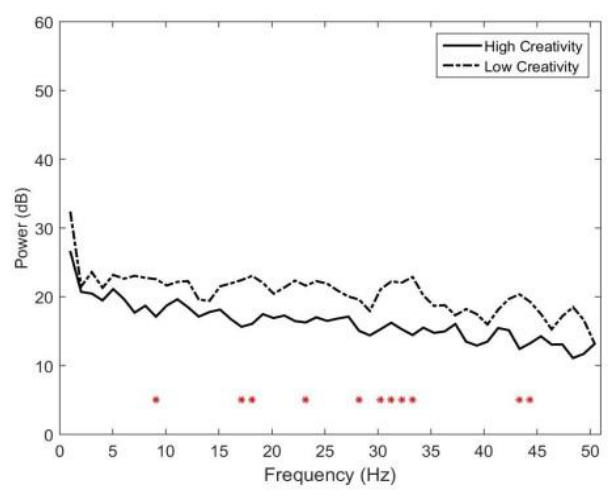

2a. Right prefrontal cluster

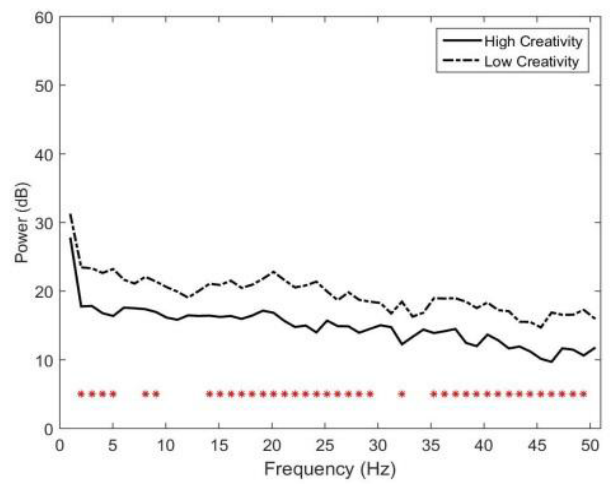

2c. Left frontoparietal cluster

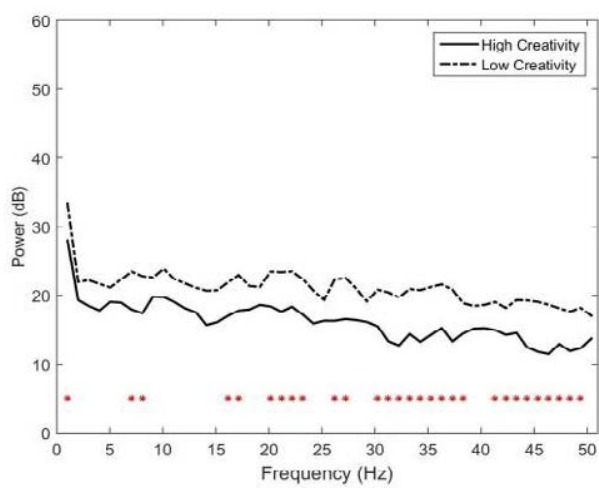

2b. Right frontotemporal cluster

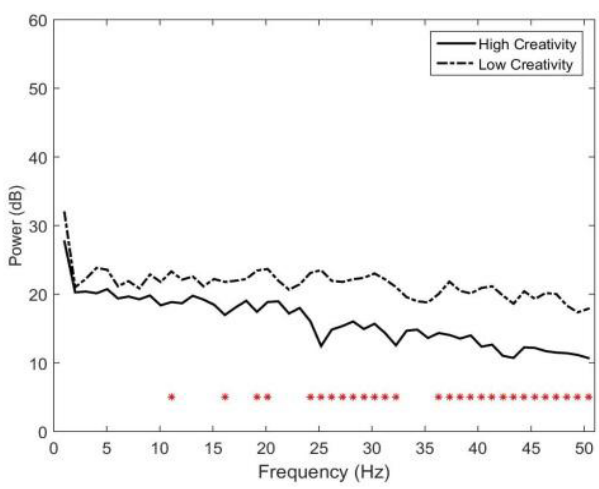

2d. Middle frontoparietal cluster

Figure 2 Spectral power and Wilcoxon signed-rank test for the realist art stimulation task.

in the distributed delta, theta, alpha, beta, and gamma bands were observed (Figure 6b). The largest differences appeared in the alpha band at $10 \mathrm{~Hz}(p=0.002079)$ and theta band at 4 $\mathrm{Hz}(p=0.004345)$ and $5 \mathrm{~Hz}(p=0.0054)$. In the middle parietal cluster (Figures $6 c$ ), only one significant power difference was observed in the high beta band at $29 \mathrm{~Hz}(p=0.012434)$. Finally, in the right temporal cluster, significant power differences in the delta, alpha, and beta bands were identified (Figures $6 \mathrm{~d}$ ). The largest differences appeared in the low gamma band at $2 \mathrm{~Hz}(p=$ $0.008463), 1 \mathrm{~Hz}(p=0.018479)$, and $11 \mathrm{~Hz}(p=0.021911)$.

The LC designers had high brain activations in the middle prefrontal and frontoparietal cortices when they engaged in the task of visual association (Figure 7a). The scalp maps and 3D dipole plots presented in Figures $\mathbf{7 b} \mathbf{b} \mathbf{7 e}$ reveal that these brain activations could be divided into four major component clusters, namely the right prefrontal, left frontotemporal, right parietal, and occipital cortices.

Our results revealed that the spectral power upon realist art stimulation was generally higher than that upon abstract art stimulation. In the right prefrontal cortex, significant power differences in the alpha, beta, and gamma bands were observed (Figures 8a). The largest differences appeared in the low gamma band at $43 \mathrm{~Hz}(p=0.002471)$ and $44 \mathrm{~Hz}(p=0.008243)$. In the left frontotemporal cluster, significant power differences were observed in the delta, theta, and beta bands (Figures $8 \mathbf{b}$ ). The largest differences appeared in the delta band at $2 \mathrm{~Hz}$
( $p=0.012161)$ and $3 \mathrm{~Hz}(p=0.033865)$. In the right parietal cluster, significant power differences were observed in the distributed delta, theta, alpha, beta, and gamma bands (Figures 8c). The largest differences appeared in the alpha band at $9 \mathrm{~Hz}$ ( $p=0.000274)$, low gamma band at $34 \mathrm{~Hz}(p=0.002751)$, and theta band at $7 \mathrm{~Hz}(p=0.003707)$. Finally, in the occipital cluster, significant power differences in the theta, beta, and gamma bands were observed (Figures 8d). The largest differences appeared in the theta band at $5 \mathrm{~Hz}(p=0.004795)$, beta band at $17 \mathrm{~Hz}(p=$ $0.007592)$, and low gamma band at $34 \mathrm{~Hz}(p=0.007592)$.

\section{Discussion}

In this study, the brain activations of the production designers increased notably in the prefrontal and parietal regions during the visual association task. The spectral power of most $\mathrm{HC}$ designers was lower than that of the LC designers, irrespective of whether realist or abstract art stimulation was provided. The differences between the $\mathrm{HC}$ and LC designers in brain activations resulting from realist art stimulation were generally greater than those in the brain activations resulting from abstract art stimulation. In the $\mathrm{HC}$ designers, brain activations resulting from abstract art stimulation were stronger than those resulting from realist art stimulation, whereas an opposite trend was observed in the LC designers.

\section{Brain activations from realist art stimulation}

Our results indicate that the right prefrontal cortex and ACC 

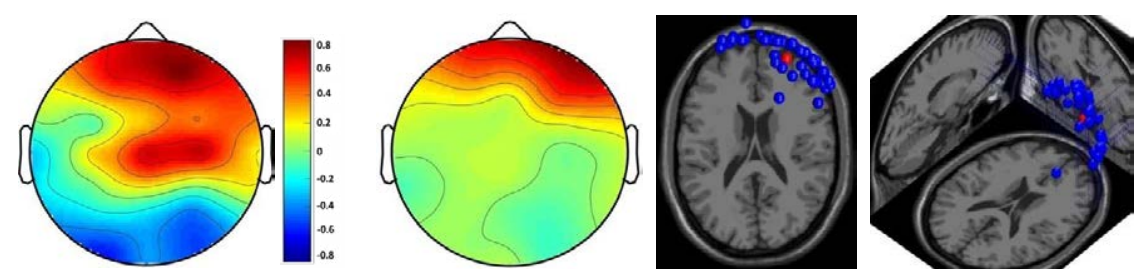

3a. Root cluster

3b. Right prefrontal cluster
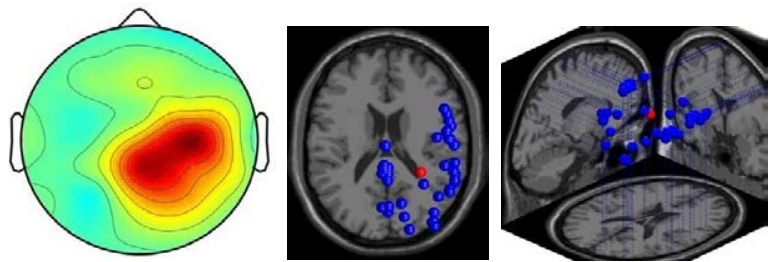

3c. Right parietal cluster
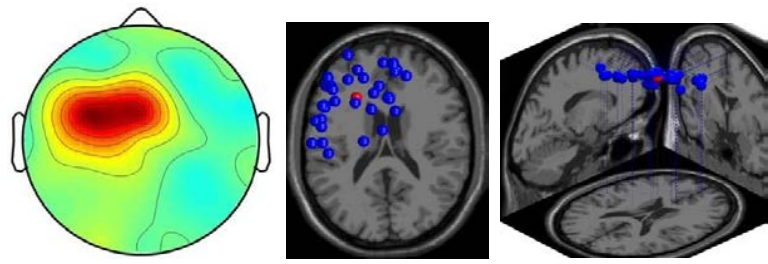

3d. Left frontoparietal cluster
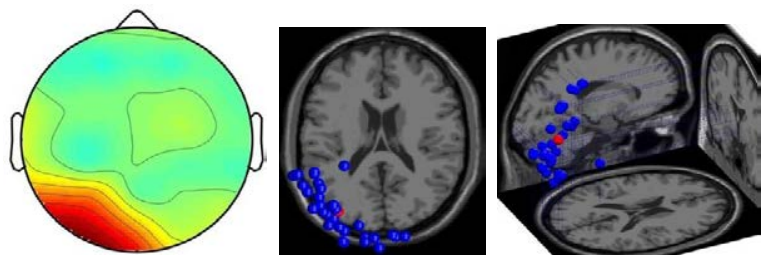

3e. Left occipital cluster

Figure 3 Scalp maps and 3D dipole plots for the abstract art stimulation results.

of the production designers were strongly activated when they engaged in the visual association task involving realist art stimulation and that the brain activations of the LC designers were stronger than were those of the $\mathrm{HC}$ designers. The right prefrontal cortex is fundamentally involved in empathy $[37,38]$, whereas the ACC controls mechanisms that detect conflict and resolves these conflicts by monitoring differential familiarity [18]. These findings are consistent with prior research $[15,16]$. They indicate that visual stimuli with increased object resolution evoked strong activation in the anterior (higher-tier) ventral regions of the production designers when they engaged in the visual association task.

The results also indicate significant differences in spectral power between the $\mathrm{HC}$ and LC designers in all component clusters. Notably, the differences in the right frontotemporal, left frontoparietal, and middle frontoparietal cortices were greater than were those in the right prefrontal cortex, particularly in the beta and gamma bands. Increases in the beta and gamma bands typically signify individuals in states of waking consciousness, alert mental activity, active thought processes, and peak luminosity in the visual field [39]. The realism art movement seeks to depict scenes cluttered with familiar objects (e.g. people, animals, and landscapes) with truth and accuracy [40], and realist artworks are usually easily understood by all social classes. Thus, we inferred that LC designers might focus on and associate realist stimuli with their daily life more easily than $\mathrm{HC}$ designers do because of visual familiarity. Our findings imply that LC designers may benefit from stimulation by representation objects, which facilitates emotional expression by prompting them to see design problems from an empathic standpoint.

\section{Brain activations from abstract art stimulation}

The EEG results indicate that the right prefrontal, right parietal, left frontoparietal, and left occipital cortices of the production designers were relatively active when they engaged in the task of visual association with Picasso's abstract art and that the brain activations of the LC designers were slightly stronger than were those of the $\mathrm{HC}$ designers. The right parietal and left frontoparietal cortices are associated with the DMN. The activation of the inferior and superior parietal cortices is associated with internally directed cognition and that of the DMN is related to a high level of self-generated thought $[6,19,41]$. In addition, the occipital cortex is fundamentally involved in visual processing, and the left hemisphere is particularly crucial for letter-string analysis [42]. The findings of the present study concur with those of previous 
studies $[15,16]$. They indicate that visual stimuli with reduced object resolution evoke strong visual association in the posterior (lower-tier) ventral regions of production designers.

In addition to showing nonsignificant differences in the right parietal cortex (Figure 4b), the results demonstrate that power differences in both the right prefrontal and left frontoparietal cortices were observed in the beta band at $20 \mathrm{~Hz}$ (Figures $4 a$ and 4c). Oscillations at $20 \mathrm{~Hz}$ appear predominantly during correct performance of a visual discrimination task [43]. Abstract representations are viewed differently from conventional representations and transcend sensory experiences accessible in everyday life [44]. This finding implies that the visual association task involving abstract art stimulation might be perceived as a visual discrimination process by LC designers because of visual unfamiliarity. Furthermore, most power differences in the left occipital cortex appeared in bands with frequencies higher than $25 \mathrm{~Hz}$ (high beta and low gamma bands) (Figure 4d). Prior research discussing the role of the left occipital cortex in visual association is limited; therefore, this topic warrants further investigation. However, numerous scholars have indicated that abstract stimuli, without a defined form to constrain the imagination of designers, are beneficial for generating original ideas [10,11]. LC designers may benefit from exposure to nonfigurative stimuli, which facilitate unusual thinking by prompting them to see design problems from a novel perspective and to build relationships between distinct domains.

\section{Brain activations in the $\mathrm{HC}$ designers}

Our results indicate that the right prefrontal, left frontotemporal, middle parietal, and right temporal cortices of the $\mathrm{HC}$ designers were strongly activated when they engaged in visual association, and that the brain activations resulting from abstract art stimulation were stronger than were those resulting from realist art stimulation. Cognitive flexibility and left frontotemporal functional connectivity during retrieval contribute to episodic memory performance [45]. Activation of the DMN (in the middle parietal cortex) is associated with self-generated thought [41]. The right temporal cortex is responsible for nonverbal memory and communication and is part of a larger network of functionally cooperative regions required for processing abstract information $[46,47]$. The findings of the present study are consistent with previous studies $[6,19,20,21]$. They indicate that the functional connectivity among these cortices could be the most critical attribute for distinguishing between $\mathrm{HC}$ and LC designers.

The results also reveal that in the right prefrontal cortex of the $\mathrm{HC}$ designers, the power spectra in the theta band resulting from abstract art stimulation was stronger than that resulting from realist art stimulation. This finding is consistent with

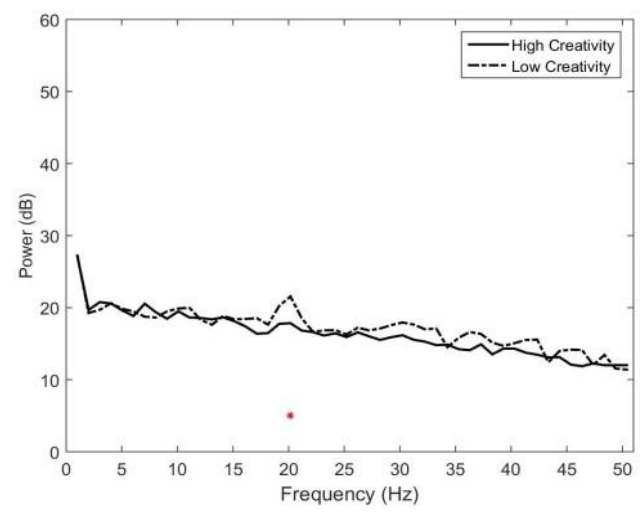

4a. Right prefrontal cluster

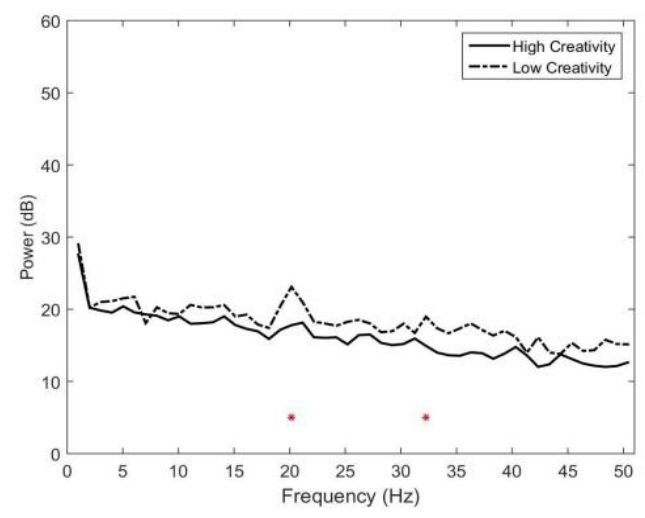

4c. Left frontoparietal cluster

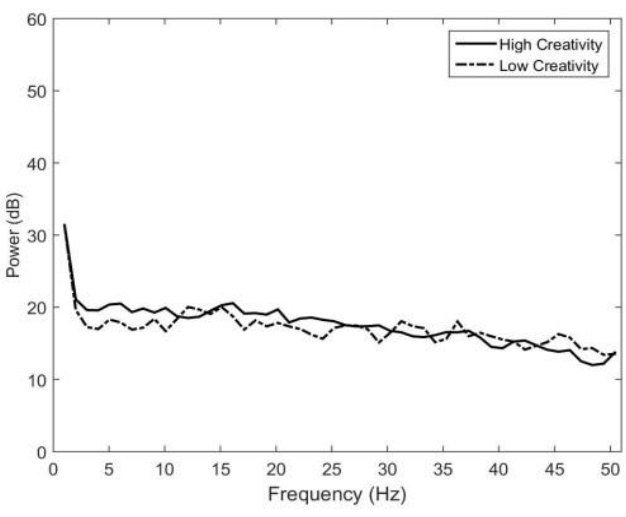

4b. Right parietal cluster

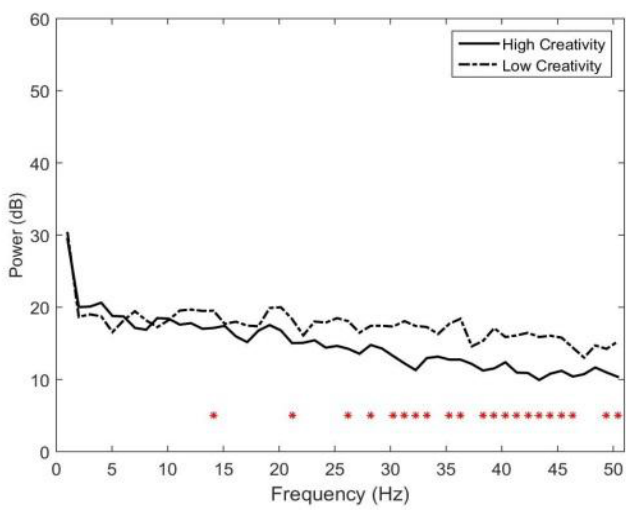

4d. Left occipital cluster

Figure 4 Spectral power and Wilcoxon signed-rank tests for abstract art stimulation. 

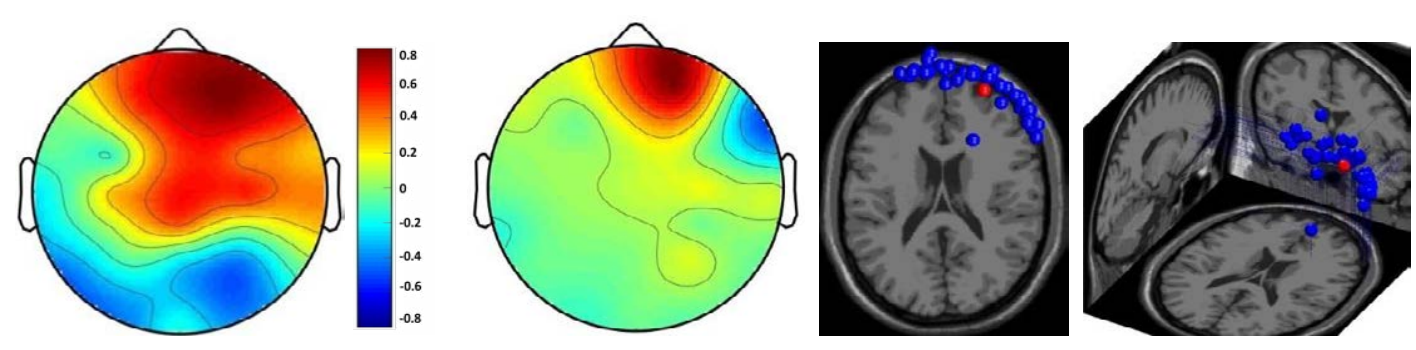

5a. Root cluster

5b. Right prefrontal cluster
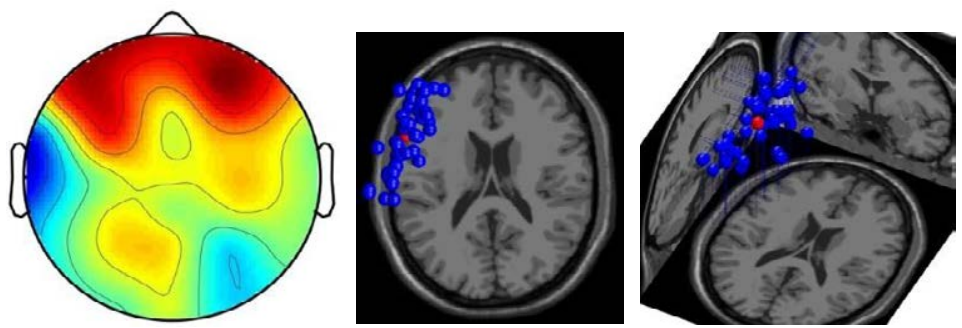

5c. Left frontotemporal cluster
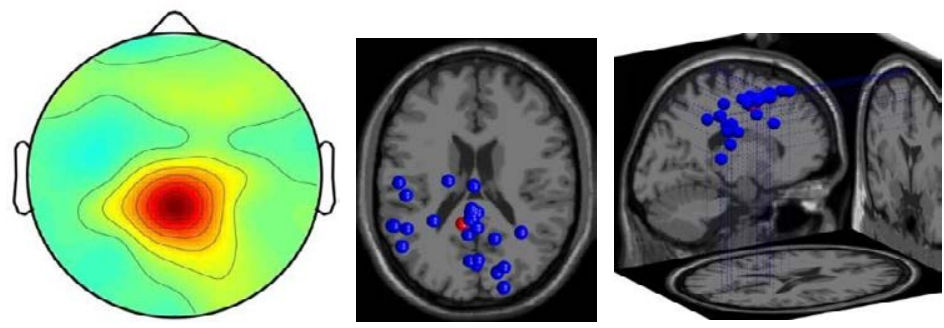

5d. Middle parietal cluster
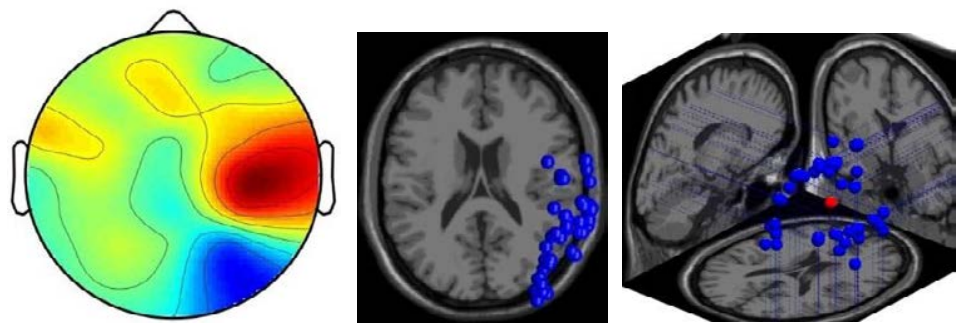

5e. Right temporal cluster

Figure 5 Scalp maps and 3D dipole plots for the HC group.

prior research $[26,48]$ and indicates that activating the right prefrontal theta band can benefit creative associations. In addition, the power differences in the left frontotemporal cortex were substantial in the theta and alpha bands. This finding is consistent with prior research $[25,45,49]$ and indicate that the visual association of the $\mathrm{HC}$ designers resulting from abstract art stimulation might be involved in reduced working memory as well as in episodic encoding and retrieval, particularly when the theta-alpha coherence was activated.

Moreover, the power differences in the middle parietal cortex appeared only in the high-beta band at $29 \mathrm{~Hz}$. This finding is consistent with prior research $[3,41]$ and indicates that visual stimulation facilitates self-generated thoughts when $\mathrm{HC}$ designers engage in visual association. Finally, the power differences in the right temporal cortex appeared primarily in bands with frequencies lower than $20 \mathrm{~Hz}$. This finding concurs with previous studies $[47,48]$ and indicates that the visual association resulting from Picasso's art stimulation is controlled by the abstract information processing network, particularly when the lowfrequency spectral power is activated. This finding also implies that LC designers may benefit from extensive access, retrieval, and application of nonverbal representations to enhance design creativity.

According to our results, the visual association of HC designers can benefit from abstract representation stimulations, which are typically distinct from visual references in the real world, with no particularly defined form to constrain the exploration of designers. In addition, theta and alpha event-related synchronizations are 


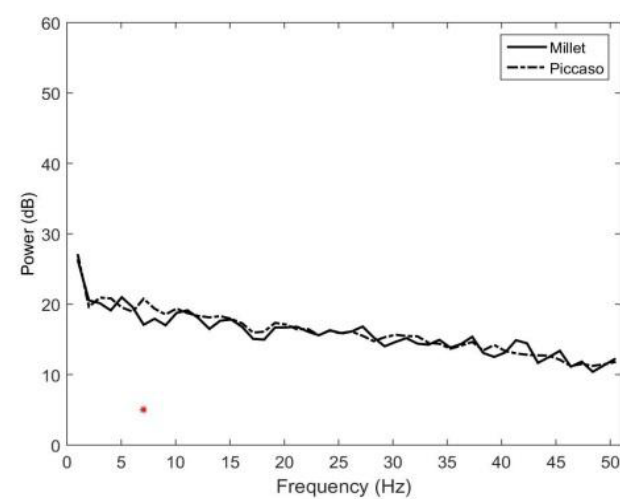

6a. Right prefrontal cluster

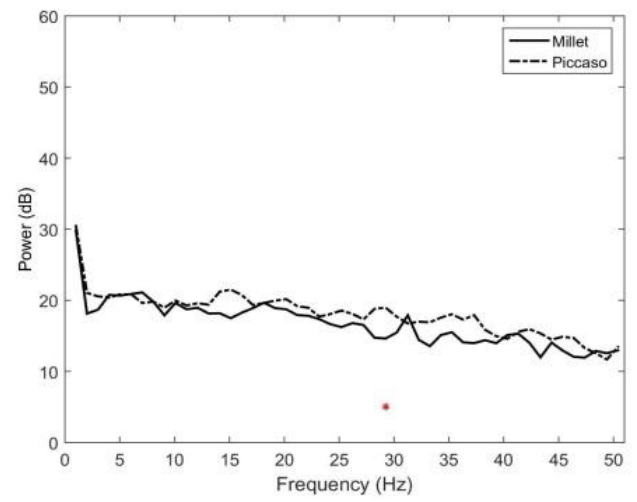

6c. Middle parietal cluster

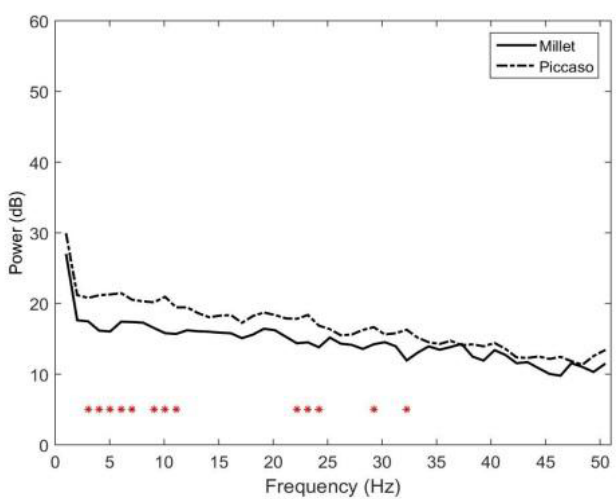

6b. Left frontotemporal cluster

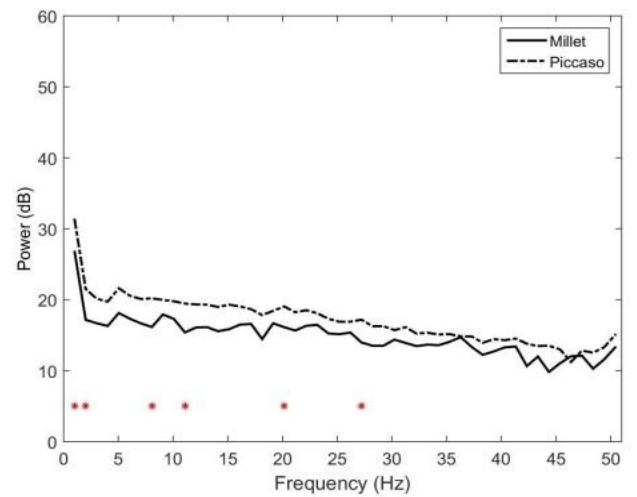

6d. Right temporal cluster

Figure 6 Spectral power and Wilcoxon signed-rank tests for the HC group.

regarded as markers of creative performance, psychological integration, and long-distance functional connectivity [30,31]. The aforementioned findings suggest that extensive theta-alpha training can enhance visual association and benefit the talent development of elite designers.

\section{Brain activations in the LC designers}

The EEG results indicate that the right prefrontal, left frontotemporal, right parietal, and occipital cortices of the LC designers were comparatively active when they engaged in visual association and that the brain activations resulting from realist art stimulation were stronger than were those resulting from abstract art stimulation. As indicated earlier, the right prefrontal cortex is involved in empathy, whereas the frontotemporal cortex is connected to episodic memory retrieval. The right parietal cortex is associated with the DMN, which is related to self-generated thought. Right parietal activation also reflects processes that are more involved in conceptual decisions on numbers than object names [50]. The occipital cortex is a visual processing centre containing most of the visual cortex. These findings indicate that the functional connectivity among these cortices highlights the pattern of LC designers' visual association.

Our results reveal that the spectral power of the LC designers in the right prefrontal cortex resulting from realist art stimulation was stronger than that from abstract art stimulation, particularly in the beta and gamma bands. In addition to providing consistent evidence regarding the facilitative role of the right prefrontal cortex in creative associations [48], the study results indicate that realist art stimulation evoked strong activation in the LC designers' visual association, but this stimulation, depicting scenes cluttered with familiar objects, might cause design fixation [51]. In addition, the power differences in the left frontotemporal cortex appeared primarily in bands with frequencies lower than $20 \mathrm{~Hz}$. This finding is consistent with prior research $[25,45,49]$ because it indicates that visual association in the LC designers resulting from realist art stimulation might be involved in episodic encoding and retrieval, particularly when theta-delta coherence is activated.

Moreover, the power differences in the right parietal cortex appeared in most frequencies, particularly in the alpha band. This experimental finding is explained by the notion that an increase in alpha power in the right parietal cortex is an indicator of the depth or elaborateness of an ongoing process of mental imagination [52]. This finding may also imply that realist art stimulation facilitated self-generated thoughts when the LC designers engaged in visual association. Finally, the power differences in the occipital cortex also appeared in most frequencies. The increased connectivity of the right parietal cortex with the occipital cortices suggests 

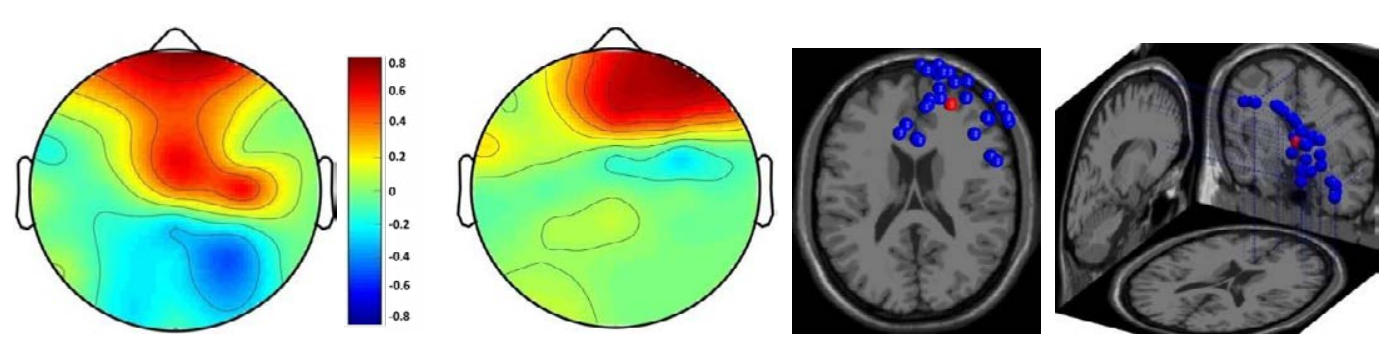

7a. Root cluster

7b. Right prefrontal cluster
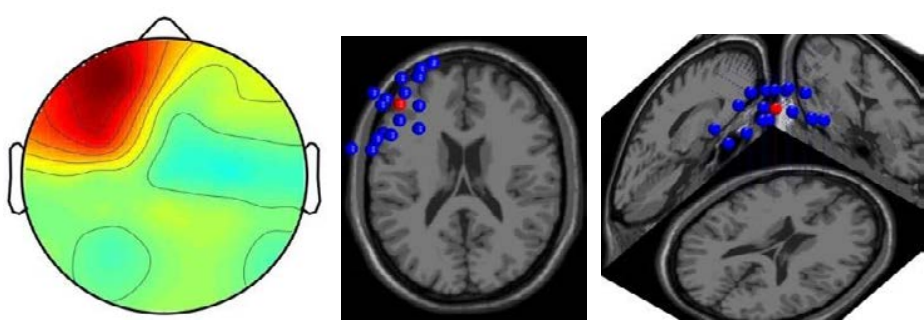

7c. Left frontotemporal cluster
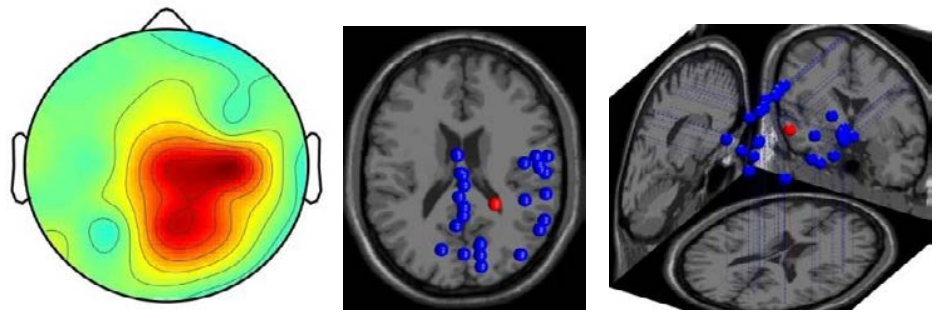

7d. Right parietal cluster
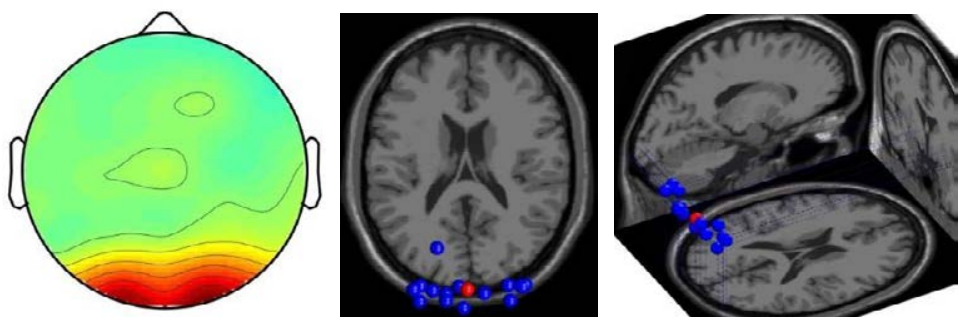

7e. Occipital cluster

Figure 7 Scalp maps and 3D dipole plots for the LC group.

an active top-down mechanism for shielding ongoing internal processes from potentially distracting sensory stimulation [41], which apparently explains the visual association process of the LC designers. This finding also implies that inappropriate and redundant sensory stimulation may hinder LC designers' visual association.

\section{Research limitations}

Neuroscience studies can provide robust evidence linking designer cognition and design practices, offering a concrete and scientific foundation for further developing research and educational strategies. However, all research has limitations. First, neuroscience studies have typically investigated simple and repeatable cognitive processes; however, designing is a complex process that requires sophisticated thinking and competence. Second, the present study was limited to the number and expertise of the participating designers. Although the designers who participated in this study were representative, 21 participants may be considered a small number for an experimental study. Third, the experimental stimuli used in this study were limited to the paintings of two artists. Additional visual representations and other forms of stimuli (i.e., text, sound, and touch) could be explored further. Fourth, EEG measures the aggregate of millions of neurons that are synchronised and thus amplify potentials. Identifying the exact location of activity in the brain through EEG is difficult because the spatial resolution is low. Finally, the wireless EEG headset used in this study was not a mature device and has considerable room for improvement in both hardware and software.

\section{Research reflections}

Despite the limitations of this study, we believe that it provides 


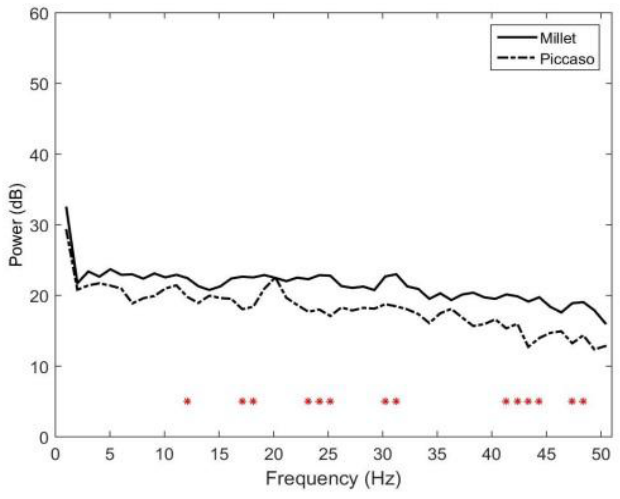

8a. Right prefrontal cluster

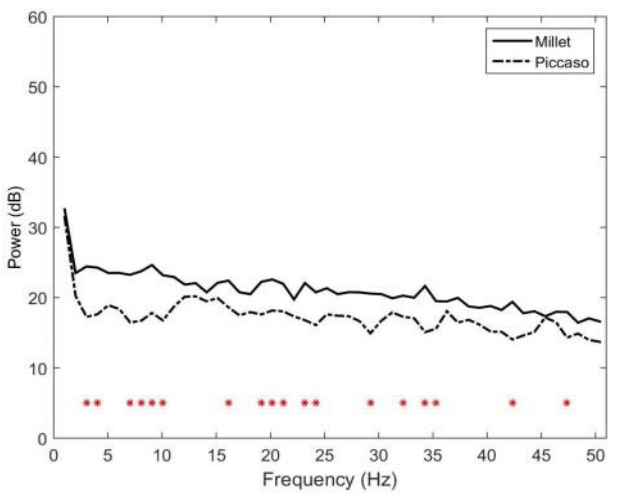

8c. Right parietal cluster

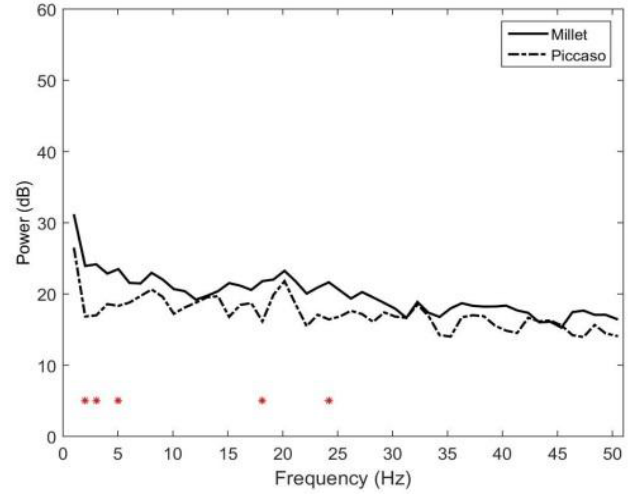

8 b. Left frontotemporal cluster

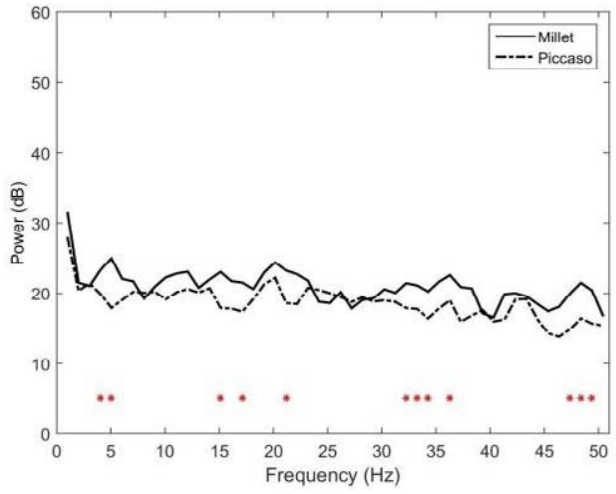

8d. Occipital cluster

Figure 8 Spectral power and Wilcoxon signed-rank tests for the LC group.

insights into the complexities of designer association, from which two notable conclusions can be drawn. First, the brain activations of production designers increased notably in the prefrontal and parietal regions when they engaged in the visual association task. Second, the spectral power of most HC designers was lower than that of the LC designers, irrespective of whether abstract or realist art stimulation was provided. Third, realist art stimulation evoked strong activation in the anterior ventral regions, whereas abstract art stimulation primarily affected the posterior regions. Fourth, the differences in the brain activations of the HC and LC designers, resulting from realist art stimulation, were generally stronger than were those resulting from abstract art stimulation. Finally, the brain activations of the $\mathrm{HC}$ designers in abstract art stimulation were stronger than were those in realist art stimulation, whereas an opposite trend was observed in the LC designers.

\section{Conclusion}

To date, little neural research has empirically explored the relationships among visual association in designers, visual stimulus types, and differences in creativity levels. Understanding these relationships can facilitate talent development in creative designers. Enhancing the creative performance of designers should be among the primary goals of design industry and education. This study elucidates a novel approach for examining the activations of specific brain regions during visual association, as well as identifying the differences elicited by distinct stimulus types in visual association processes among designers with different levels of creativity. Our study provides further support for integrated neural theories of association, visual stimulation, and designer creativity. It should serve as a gateway for further investigation into the role of visual association in creative performance. Thus, we are optimistic that the approach described in our study constitutes at least the first step towards a broad understanding of the neural basis of design thinking and behaviour.

\section{Acknowledgements}

The current study is part of the research project (MOST 104-2511-S-002-008-MY2) supported by Taiwan's Ministry of Science and Technology. The authors would like to extend their gratitude to the insightful suggestions of anonymous Journal of Neurology \& Neuroscience reviewers and the Brain Rhythm Incorporation. 


\section{References}

1 Crilly N (2010) The structure of design revolutions: Kuhnian paradigm shifts in creative problem solving. Design Issues 26: 54-66.

2 Seitamaa HP, Huotilainen M, Makela M, Groth C, Hakkarainen K (2016) How can neuroscience help understand design and craft activity? The promise of cognitive neuroscience in design studies. For Maka Demisk 9: 1-16.

3 Yao SN, Lin CT, King JT, Liu YC, Liang C (2017) Learning in the visual association of novice and expert designers. Cognitive Systems Research 43: 76-88.

4 Goldschmidt G (2015) Ubiquitous serendipity: Potential visual design stimuli are everywhere. In Gero JS (Ed), Studying visual and spatial reasoning for design creativity. Houten, Netherlands: Springer pp. 205-214.

5 Afsaneh N, Parisa D, Karim A, Saleh G (2015) The relationship between visual associations of a random fixed cardboard piece and introversion-extroversion as well as the level of creativity in a group of visual artists. Motaleate Tatbighi Honar 5: 25-34.

6 Liang C, Lin CT, Yao SN, Chang WS, Liu YC, et al. (2017) Visual attention and association: An electroencephalography study in expert designers. Design Studies 48: 76-95.

7 Gazzaley A, Rissman J, Cooney J, Rutman A, Seibert T, et al. (2007) Functional interactions between prefrontal and visual association cortex contribute to top-down modulation of visual processing. Cerebral Cortex 17: i125-i135.

8 Grace K, Saunders R, Gero J (2011) Interpretation-driven visual association. In: Ventura D, Gervas P, Harrell DF, Maher ML, Pease A Wiggins $G$ (eds), Proceedings of the Second International Conference on Computational Creativity. Mexico City, Mexico. pp. 132-134.

9 Popovic V (2004) Expertise development in product design: Strategic and domain-specific knowledge connections. Design Studies 25: 527-545.

10 Cila N, Hekkert P, Visch V (2014) Source selection in product metaphor generation: The effects of salience and relatedness. International Journal of Design 8: 15-28.

11 Zahner D, Nickerson JV, Tversky B, Corter JE, Ma J (2010) A fix for fixation? Re-representing and abstracting as creative processes in the design of information systems. Artif Intell Eng Des Anal Manuf 24: 231-244.

12 Goldschmidt G, Smolkov M (2006) Variances in the impact of visual stimuli on design problem solving performance. Design Studies 27 549-569.

13 Cela-Conde CJ, Marty G, Maestu F, Ortiz T, Munar E, et al. (2004) Activation of the prefrontal cortex in the human visual aesthetic perception. Proceeding of the National Academy of Sciences of the USA (PNAS) 101: 6321-6325

14 Silveira S, Graupmann V, Frey D, Blautzik J, Meindl T, et al. (2012) Matching reality in the arts: Self-referential neural. Percetion 41: 569-576.

15 Fairhall SL, Ishai A (2008) Neural correlates of object indeterminacy in art compositions. Conscious Cogn 17: 923-932.

16 Mechelli A, Price CJ, Friston KJ, Ishai A (2004) Where bottom-up meets top-down: neuronal interactions during perception and imagery. Cerebral Cortex 14: 1256-1265.

17 Andreasen NC (2011) A journey into chaos: Creativity and the unconscious. Mens Sana Monogr 9(1), 42-53.
18 Bunge SA, Burrows B, Wagner AD (2004) Prefrontal and hippocampal contributions to visual associative recognition: Interactions between cognitive control and episodic retrieval. Brain Cogn 56: 141-152.

19 Kurth-Nelson Z, Barnes G, Sejdinovic D, Dolan R, Dayan P (2015) Temporal structure in associative retrieval. E-Life 4: e04919.

20 Buckner RL, Andrews-Hanna JR, Schacter DL (2008) The brain's default network: Anatomy, function and relevance to disease. Ann N Y Acad Sci 1124: 1-38.

21 Schacter DL, Addis DR, Hassabis D, Martin VC, Spreng RN, et al. (2012) The future of memory: Remembering, imagining and the brain. Neuron 76: 677-694.

22 Gerlach KD, Spreng RN, Gilmore AW, Schacter DL (2011) Solving future problems: Default network and executive activity associated with goal-directed mental simulations. Neurolmage 55: 1816-1824.

23 Szpunar KK, St. Jacques PL, Robbins CA, Wig GS, Schacter DL (2014) Repetition-related reductions in neural activity reveal component processes of mental simulation. Scan 9: 712-722.

24 Bhattacharya J, Petsche H (2005) Drawing on mind's canvas: Differences in cortical integration patterns between artists and nonartists. Human Brain Mapping 26: 1-14.

25 Sviderskaya NE, Taratynova GV, Kozhedub RG (2006) The effects of the experience of forming visual images on the spatial organization of the EEG. Neurosci Behav Physiol 36: 941-949.

26 Volf NV, Tarasova IV (2010) The relationships between EEG $\theta$ and $\beta$ oscillations and the level of creativity. Human Physiology 36: 132-138.

27 Jausovec N (2000) Differences in cognitive processes between gifted, intelligent, creative, and average individuals while solving complex problems: An EEG study. Intelligence 28: 213-237.

28 Fink A, Graif B, Neubauer AC (2009) Brain correlates underlying creative thinking: EEG alpha activity in professional vs. novice dancers. Neuro Image 46: 854-862.

29 Sviderskaya NE (2011) Influence of informational oversaturation on the quality of creative activity and spatial organization of EEG. Human Physiology 37: 667-672.

30 Gruzelier J (2008) A theory of alpha/theta neurofeedback, creative performance enhancement, long distance functional connectivity and psychological integration. Cogn Process 10: 101-109.

31 Gruzelier JH, Thompson T, Redding E, Brandt R, Steffert T (2014) Application of alpha/theta neurofeedback and heart rate variability training to young contemporary dancers: State anxiety and creativity: Int J Psychophysiol 93: 105-111.

32 Gough HG (1979) A creative personality scale for the adjective checklist. J Pers Soc Psychol 37: 1398-1405.

33 Onton J, Westerfield M, Townsend J, Makeig S (2006) Imaging human EEG dynamics using independnt component analysis. Neurosci Biobehav Rev 30: 808-822.

34 Cantero JL, Atienza M, Gómez CM, Salas RM (1999) Spectral structure and brain mapping of human alpha activities in different arousal states. Neuropsychobiology 39: 110-116.

35 Grützner C, Wibral M, Sun M, Rivolta D, Singer W, et al. (2013) Deficits in high- $(>60 \mathrm{~Hz})$ gamma-band oscillations during visual processing in schizophrenia. Front Hum Neurosci 7: 88.

36 Tang Y, Chorlian DB, Rangaswamy M, Porjesz B, Bauer L, et al. (2007) Genetic influences on bipolar EEG power spectra. IInt J Psychophysiol 65: 2-9. 
37 Gallup GGJ, Platek SM (2002) Cognitive empathy presupposes selfawareness: Evidence from phylogeny, ontogeny, neuropsychology and mental illness. Behav Brain Sci 25: 36-37.

38 Ochsner KN, Silvers JA, Buhle JT (2012) Functional imaging studies of emotion regulation: A synthetic review and evolving model of the cognitive control of emotion. Ann N Y Acad Sci 1251: E1-E24.

39 Bauer M, Stenner MP, Friston KJ, Dolan RJ (2014) Attentional modulation of alpha/beta and gamma oscillations reflect functionally distinct processes. J Neurosci 34: 16117-16125.

40 Finocchio R (2000) Nineteenth-century French realism. In: Heilbrunn timeline of art history. The Metropolitan Museum of Art, New York.

41 Benedek M, Jauk E, Beaty RE, Fink A, Koschutnig K, et al. (2016) Brain mechanisms associated with internally directed attention and selfgenerated thought. Scientific Reports 6: 22959.

42 Cohen L, Lehericy S, Chochon F, Lemer C, Rivaud S, et al. (2002) Language-specific tuning of visual cortex? Functional properties of the visual word form area. Brain 125: 1054-1069.

43 Wróbel A (2000) Beta activity: A carrier for visual attention. Acta Neurobiologiae Experimentalis 60: 247-260.

44 Silveira S (2014) The influence of mental frames on the neurocognitive processing of visual art. Doctoral dissertation of Ludwig-MaximiliansUniversität. Munich, Germany: Ludwig-Maximilians-Universität.

45 Blankenship TL, Bell MA (2015) Frontotemporal coherence and executive functions contribute to episodic memory during middle childhood. Dev Neuropsychol 40: 430-444.

46 Liu YC, Liang C (2017) Investigating how the brain activations of visual attention differ among designers with different levels of creativity. In New developments in visual attention research. Hauppauge, NY: Nova Science Publishers.

47 Wisniewski I, Wendling AS, Manning L, Steinhoff BJ (2012) Visuospatial memory tests in right temporal lobe epilepsy foci: Clinical validity. Epilepsy and Behavior 23: 254-260.

48 Kowatari Y, Lee SH, Yamamura H, Nagamori Y, Levy P, et al. (2009) Neural networks involved in artistic creativity. Human Brain Mapping 30: $1678-1690$.

49 Heimans JJ, Reijneveld JC, Stam CJ (2013) Magneto-encephalography, functional connectivity, and neural network topology in diffuse lowgrade gliomas. In: Diffuse low-grade gliomas in adults. Springer, London. pp. 321-336.

50 Cappelletti M, Lee HL, Freeman ED, Price CJ (2010) The role of right and left parietal lobes in the conceptual processing of numbers. Cognitive Neurosci 22: 331-346.

51 Cardoso C, Badke-Schaub P (2011) The influence of different pictorial representations during idea generation. j Creat Behav 45: 130-146.

52 Von Stein A, Sarnthein J (2000) Different frequencies for different scales of cortical integration: From local gamma to long range alpha/ theta synchronization. Int J Psychophysiol 38: 301-313. 